\title{
A Theory for the Comparative RF Surface Fields at Destructive Breakdown for Various Metals
}

\section{Introduction}

By destructive breakdown we mean a breakdown event that results in surface melting over large areas on the iris tip region of an accelerator structure. The melting is the result of the formation of macroscopic areas of plasma in contact with the surface. The plasma bombards the surface with an intense ion current $\left(\sim 10^{8} \mathrm{~A} / \mathrm{cm}^{2}\right)$, which is equivalent to a pressure on the order of a thousand Atmospheres. A radial gradient in the pressure produces a ponderomotive force that causes molten copper to migrate away from the iris tip, resulting in a measurable change in the iris shape. This distortion in the iris shape in turn produces an error in the cell-to-cell phase shift of the accelerating wave with a consequent loss in synchronism with the electron beam and a reduction in the effective accelerating gradient. Assuming a long lifetime is desired for the structure, such breakdowns must be avoided or at least limited in number. The accelerating gradient at which these breakdowns begin to occur imposes, therefore, an absolute limit on an operationally attainable gradient.

The destructive breakdown limit (DBL) on the accelerating gradient depends on a number of factors, such as the geometry of the irises and coupler, the accuracy of the cell-to-cell tuning ("field flatness"), and the properties of the metal used in the high E-field regions of the structure. In this note we consider only the question of the dependence of the DBL on the metal used in the high surface field areas of the structure.

There are also various types of non-destructive breakdowns (NDB's) that occur during the "processing" period that, after the initial application of high power, is necessary to bring the gradient up to the desired operating level. During this period, as the input power and gradient are gradually increased, thousands of such NDB's occur. These breakdowns produce a collapse in the fields in the structure as energy stored in the fields is absorbed at the breakdown site. They are often marked by vacuum bursts and an increase in power reflected from the structure. The usual cause for NDB's during processing is the "explosion" of field emitters at sharp geometrical features on the metal surface. Exposed impurities in the metal surface can also produce NDB's as they are "burned" off by H-field heating or explosive field emission.

The breakdown process can be roughly divided into four stages: (1) the formation of "plasma spots" at field emission sites, each spot leaving a crater-like footprint; (2) crater clustering, and the formation of areas with hundreds of overlapping craters; (3) surface melting in the region of a crater cluster; (4) the process after surface melting that leads to destructive breakdown.

\section{Plasma spot formation}

As the gradient in an accelerating structure is increased after the initial application of high power, sharp geometrical features in high field regions (near the iris inner radius) will begin to field-emit. Following the theory under development here, at some level of field emission current the tip of a sharp feature will begin to melt. The radius of curvature of the liquid tip is determined by the equilibrium between the pull of the $\mathrm{E}^{2}$ force and the restraining force of surface tension. As the field is increased further, the radius of the tip will decrease until, at a critical radius an instability causes the tip to literally explode, emitting a jet of liquid metal 
micro-droplets. The field emission current quickly vaporizes the micro-droplets and ionizes the resulting metal gas, leading to the formation of a plasma at the emitter site with a diameter on the order of a few microns. The plasma forms a Debye sheath where the plasma is in contact with the metal. The sheath is essentially a space charge limited Child's law diode, injecting electrons into the plasma and bombarding the metal surface with an intense ion counter-current on the order of $10^{8} \mathrm{~A} / \mathrm{cm}^{2}$. The metal below the spot melts on a sub-nanosecond time scale. Both the molten area and associated plasma spot expand until the plasma quenches after some tens on nanoseconds. A small crater-like feature with a diameter of 5-20 microns is left behind. The physics of the formation of plasma spots (called cathode spots in the literature on DC vacuum arcs) is essentially identical for both DC and RF fields. The craters left behind are indistinguishable.

The energy from ion bombardment at the plasma sheath that goes into creating a crater is very small-only about $1 \times 10^{-5} \mathrm{~J}$. However, the electrons injected into the vacuum by the plasma spot ( 10 A) pick up several hundreds of $\mathrm{keV}$ in energy from the RF field. The energy extracted from the field in $10 \mathrm{~ns}$ is on the order of $0.03 \mathrm{~J}$. This is enough to collapse the field in a cell of a typical standing-wave structure, producing RF reflected power and tripping off the klystron powering the structure. In a typical traveling-wave structure, energy flows into the cell rapidly enough to prevent field collapse. However, there is often a vacuum burst associated with the formation of the plasma spot, which can also trip off the RF source. In any case, the formation of a single crater some ten microns across has a completely negligible effect on the measurable RF properties of the structure.

\section{Crater clustering}

The crater left behind by the destruction of a field emitter looks somewhat like a volcano crater with material thrown out from the interior of the crater forming a jagged rim surrounding it. These sharply-pointed rim features can themselves become field emitters. As the sharpest single features are burned away, it becomes more and more likely that new plasma spots will form on the rim of an existing crater, producing two overlapping craters. The total rim circumference of the two overlapping craters is larger than the rim circumference of a single crater, making the probability higher that an additional plasma spot will be added to this feature, rather than at the rim of an isolated crater. In this way clusters of hundreds of overlapping craters can form. Conditions are now ripe for dozens of closely spaced plasma spots to be alive at once during a time window of $30 \mathrm{~ns}$ or so-the lifetime of a single spot. Back-bombardment from the electrons emitted by these spots that return to impact the surface can now heat the entire crater cluster area and raise the surface temperature to the melting point. The physics of this process is outlined in the next section.

\section{Conditions for surface melting}

As mentioned above, a plasma spot in contact with a metal surface is essentially a space charge limited cathode, obeying Child's Law, that emits $\sim 10$ A of $\sim 20 \mathrm{eV}$ electrons into the vacuum. Electrons emitted in the 90 degree phase period after the peak of the RF field will return to impact the surface within a radius on the order of 100 microns at an RF frequency of $10 \mathrm{GHz}$ (this radius is proportional to the RF wavelength). For a large crater cluster with many spots alive at the same time, the areas heated by the individual spots will overlap. The energy spectrum of these bombarding electrons ranges from zero to a peak of several hundred keV. To calculate the surface heating, it is also necessary to know the number of electrons as a function of energy. A crude calculation of the power spectrum of the impacting electrons gives an average impact energy on the order of $50 \mathrm{keV}$ (this calculation can easily be refined). Based on the preceding information, a rough calculation gives a heating power per unit area of $\mathrm{P}_{\mathrm{A}} \sim 10^{8} \mathrm{~W} / \mathrm{cm}^{2}$. 
However, in this note we are only interested in the dependence of the breakdown gradient on the material used to fabricate the iris tips and an absolute value for $\mathrm{P}_{\mathrm{A}}$ is not needed.

The heat equation has a simple solution for the surface temperature as a function of time if the incident power is applied exactly at the surface of a semi-infinite slab. In our case, however, the incident electrons penetrate into the metal by a distance that depends on their energy. A single $\mathrm{keV}$-energy electron produces a shower, with an intensity (power dissipated per unit depth) that increases to a maximum at a depth dependent on the material and the incident electron energy. The shower intensity then trails off to zero with increasing depth. An examination of the literature shows that the shape of the intensity plot is roughly independent of material and the depth at the shower maximum scales simply as $1 / \rho$, where $\rho$ is the density of the metal. In the energy range of interest, the penetration depth varies with impact energy $\mathrm{E}$ as $\mathrm{E}^{1.5}$. For $\mathrm{E}$ in $\mathrm{keV}$ and $\rho$ in $\mathrm{gm} / \mathrm{cm}^{3}$, the penetration depth is given by

$$
\mathrm{X}_{\mathrm{P}}(\mu \mathrm{m})=0.07 \mathrm{E}^{1.5} / \rho
$$

A second parameter needed to construct an approximate solution of the heat equation is the diffusion depth, $\mathrm{X}_{\mathrm{D}}$, at time $\mathrm{t}$ :

$$
\mathrm{X}_{\mathrm{D}}(\mu \mathrm{m})=1 \times 10^{4}(\mathrm{Dt})^{1 / 2}
$$

where $\mathrm{D}$ is the diffusivity

$$
\mathrm{D}\left(\mathrm{cm}^{2} / \mathrm{sec}\right)=\mathrm{K}_{\mathrm{th}} / \rho \mathrm{C}_{\mathrm{s}}
$$

Here $\mathrm{K}_{\mathrm{th}}$ is the thermal conductivity and $\mathrm{C}_{\mathrm{S}}$ is the specific heat.

For a semi-infinite slab with heat applied to the surface, the temperate rise at time $t$ is

$$
\Delta \mathrm{T}=\left(2 \mathrm{P}_{\mathrm{A}} / \pi^{1 / 2} \mathrm{~K}_{\mathrm{th}}\right)(\mathrm{Dt})^{1 / 2} \text {. }
$$

We will assume that the metal surface is brought to the melting point in a very short time- $30 \mathrm{~ns}$ (the reason for this choice will be explained later). At $30 \mathrm{~ns}$ we find for all the metals considered that the penetration depth is large compared to the diffusion depth. In this case, an approximate solution of the heat equation can be formed by considering $X_{D}$ as the "surface" and that the heat, $\mathrm{P}_{\mathrm{A}}\left(\mathrm{X}_{\mathrm{D}} / \mathrm{X}_{\mathrm{P}}\right)$, dissipated there drives the temperature rise given by Eq. (4). In this simple approximation we've also assumed that heat is dissipated uniformly over the penetration depth. A more rigorous calculation is in progress.

The temperature rise normalized to the melting point, $\mathrm{T}_{\mathrm{m}}$, at a fixed time is

$$
\left(\Delta \mathrm{T} / \mathrm{T}_{\mathrm{m}}\right) \sim \mathrm{P}_{\mathrm{A}}\left(\mathrm{X}_{\mathrm{D}} / \mathrm{X}_{\mathrm{P}}\right)\left(\mathrm{X}_{\mathrm{D}} / \mathrm{K}_{\mathrm{th}} \mathrm{T}_{\mathrm{m}}\right)
$$

At the melting point $\Delta \mathrm{T}=\mathrm{T}_{\mathrm{m}}$. Using Eqs. (1) - (4), we find that at the melting point $\mathrm{P}_{\mathrm{A}} \sim \mathrm{T}_{\mathrm{m}} \mathrm{C}_{\mathrm{S}}$. Both the density and the thermal conductivity have cancelled out. Since the surface E-field is proportional to $\left(\mathrm{P}_{\mathrm{A}}\right)^{1 / 2}$, we obtain finally that the surface field at breakdown scales as

$$
\mathrm{E}_{\mathrm{S}} \sim\left(\mathrm{T}_{\mathrm{m}} \mathrm{C}_{\mathrm{s}}\right)^{1 / 2}
$$

The scaling above becomes even simpler using the Debye Approximation for the specific heat of metals [see, for example, F. Reif, Fundamentals of statistical and thermal physics (McGraw- 
Hill, NY, 1965) pp. 411-417]. The theory shows that the specific heat can be approximated reasonably well at room temperature and above by

$$
\mathrm{C}_{\mathrm{s}}\left(\mathrm{J} / \mathrm{g}-{ }^{\circ} \mathrm{C}\right) \approx 25 / \mathrm{A}
$$

where $\underline{\mathrm{A}}$ is the atomic weight of the metal (for certain materials having a high "Debye temperature", a small correction to the constant in this expression is necessary). The scaling of the breakdown field with iris tip material then becomes simply

$$
\mathrm{E}_{\mathrm{S}} \sim\left(\mathrm{T}_{\mathrm{m}} / \mathrm{A}\right)^{1 / 2}
$$

However, in obtaining values for the relative breakdown fields of various metals we will use Eq. (5). Equation (6) does tell us that we'll do better by looking at metals with low atomic weights.

By normalizing Eq. (5) so that the relative breakdown field for copper is unity, we obtain a figure of merit for various materials defined by

$$
\begin{aligned}
& \mathrm{FM}=4.9 \times 10^{-2}\left(\mathrm{~T}_{\mathrm{m}} \mathrm{C}_{\mathrm{s}}\right)^{1 / 2} \\
& \mathrm{FM}(\mathrm{Cu})=1
\end{aligned}
$$

Almost all the reasonable metals in the periodic table have been examined for their Figures of Merit. A table attached to this note shows the results. The FM's are separated into seven groups. The first group contains metals with FM's less than 1 (worse than copper). Note that gold is the worst of the lot - very high $\underline{\mathrm{A}}$ and not a very high melting point. These metals are given a failing grade. The next group contains metals that are only slightly better than copper. They get a D grade. Somewhat more interesting are the metals with FM's in the range 1.1 to 1.2. We'll give them a C. Quite a bit more interesting are the metals with FM's in the range 1.2 to 1.3. This group, which includes niobium, moly and stainless steel, gets a B. The group consisting of chromium, titanium, scandium and vanadium is very interesting, with FM's in the range 1.4 to 1.5. This group gets an A grade. The superstar metal is beryllium (very low $\underline{A}$ and a reasonably high melting point), which has an $\mathrm{FM}=2.37$. Also shown in the table is a group of "wild cards": boron, silicon and carbon. The first two are insulators, while carbon has a resistivity about 600 times that of copper. The FM for carbon is almost as high as that for beryllium. Possibly it could be sputtered or in some way coated onto an iris tip. If so, with its very high melting point (about $3500{ }^{\circ} \mathrm{C}$ ), it would make the ideal iris-tip material. Silicon is also an interesting material, with an extensive technology for coating it on metals.

A second attachment shows the periodic table of the elements with the FM's color-coded on it. From Eq. (6) the FM is proportional to $\left(\mathrm{T}_{\mathrm{M}} / \mathrm{A}\right)^{1 / 2}$, so the FM's tend to increase as we go upward in the table to elements with smaller atomic weights. Within one period, the best FM's tend to cluster in the center, flanked on either side by elements with lower FM's. There is also a left-right asymmetry, with higher FM metals on the left. Since $\underline{A}$ is reasonably constant along a given row in the table, these features can only be explained by the variation in melting temperature. The third attachment shows the melting points of the metals in periods 4,5 and 6 . The variation in the FM along a given row of the periodic table is explained by these melting point plots. There is also a theory for the melting point of a metal, in which the melting point is expressed in terms of the atomic weight, the density and the Debye temperature. 


\section{From surface melting to destructive breakdown}

We consider the parameters for the proposed NLC (Next Linear Collider) project, studied at SLAC and at KEK in Japan for about a decade. The NLC was based on the use of roomtemperature copper accelerator structures; it would have produced a c.m. energy of $1 \mathrm{GeV}$. Unfortunately, the NLC project is now defunct, an international committee having chosen a machine based on superconducting cavities as the collider that will actually be built. However, as we show in this note, materials other than copper have the potential to make possible accelerating gradients at least double that of copper. This would cut the accelerator length at least in half, resulting in a considerable reduction in cost. Since the actual construction of this multibillion dollar international machine is still many years away, a higher gradient room-temperature accelerator structure technology might yet prove attractive for the final design. In any case, an accelerator structure capable of a gradient on the order of $150 \mathrm{MV} / \mathrm{m}$ would make a post-ILC 3 $\mathrm{TeV}$ collider much more viable.

The NLC design used an RF pulse length of $400 \mathrm{~ns}$. In our theory here, we propose that for the major fraction of the pulse length the surface in a crater cluster region, heated by electron bombardment from dozens of closely spaced plasma spots, is in the molten state. During this time electro-mechanical forces act on the liquid surface to gradually form (over hundreds or thousands of pulses) sharp geometric features, following the process outlined below. To maximize the time for these forces to act on the molten surface, the surface metal should be raised to the melting point in a small fraction of the total pulse length. This is the reason for the choice of $30 \mathrm{~ns}$ in the preceding section for the melting time.

Figure 1 shows the geometric features formed by exposing a thin layer of molten metal to a dc electric field. We propose that similar features also form when an rf field acts over many pulses on the liquid surface layer produced by back-bombardment heating in a cluster of plasma spots. These features follow a somewhat regular pattern. Many of them have a roughly conical base with a vertical column or jet emerging from the apex. The sides of the cones make a roughly $45^{\circ}$ angle with respect to the base. The projections emerging from the tips of the cones all have roughly the same diameter. This is indicative of the onset of an instability at a certain apex radius. In the following, we develop a model based on this shape.

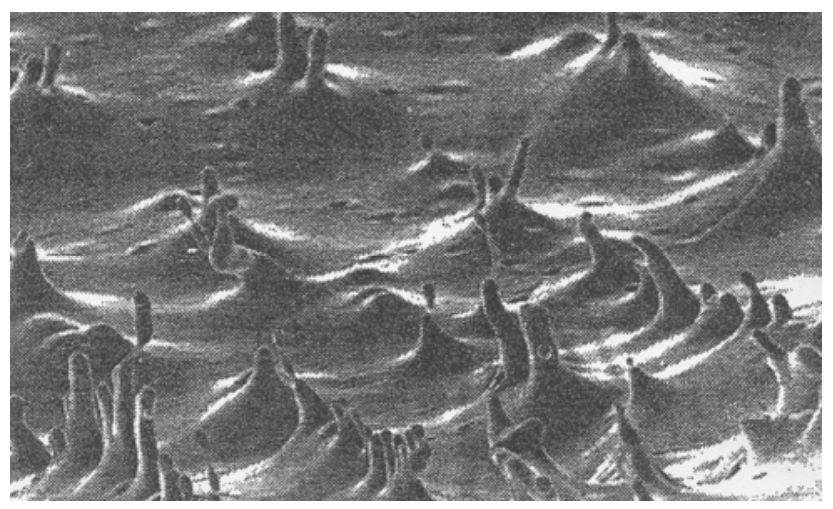

Figure 1: Surface features after action of an electric field on a thin layer of molten metal on a planar electrode [G.A. Mesyats, Explosive electron emission (URO-Press. Ekaterinburg, 1998) p. 29].

The model first assumes that the back-bombarding electrons produce sufficient heating to melt a thin layer of the surface in $30 \mathrm{~ns}$ or so at the beginning of each rf pulse, cooling and solidifying between pulses. Since the molten material cannot move very far in one rf pulse, 
geometric features with a scale of tens of microns must develop over hundreds or even thousands of pulses. Next assume that there are random height perturbations on the liquid surface, and that these can be modeled as a portion of a spherical surface with radius $r_{1}$ (see Fig.2). This radius is given by the balance between the $\mathrm{E}^{2}$ force pulling on the surface and the force due to the surface tension of the liquid metal ( $\alpha=1.3 \mathrm{Nt} / \mathrm{m}$ for copper). It is given by

$$
r_{1}=8 \alpha / \varepsilon_{0} E_{S}^{2}
$$

where the surface field $E_{S}$ is approximately equal to the unperturbed electric field, $E_{0}$, at the surface. The scale of these perturbations is set by Eq. (8), where $E_{S}$ is given by the unperturbed field. The field will actually be slightly enhanced at the surface of the perturbation causing it to grow higher by the process outlined below. This in turn enhances the field still more, etc. Following the shape of the surface projections suggested by Fig. 1, we model the growing perturbation as a conical pyramid with sides making angle $\phi$ with respect to the base. We assume that the cone is capped by a segment of a sphere with radius $r$, as show in Fig. 2. We assume that the analytic part of the growth process, where the cap radius is set by the condition for hydrostatic equilibrium, Eq. (8), starts with the initial spherical segment of radius $r_{1}$. As the height of the cone increases, the radius of the cap decreases and the surface field $E_{S}$ and enhancement factor $\beta=E_{S} / E_{0}$ also increases. Simulations show that beta can be modeled as $\beta \sim$ $\mathrm{r}^{-\mathrm{n}}$, where $\mathrm{n}$ is a function of the base angle $\phi$. For the molten cap to be in hydrostatic equilibrium, the radius must vary as $r / r_{1}=E_{1}^{2} / E_{S}^{2}$, giving $\beta=\beta_{1}\left(r / r_{1}\right)^{-1 / 2}$, where $\beta_{1}$ is the value of beta at $r=$ $\mathrm{r}_{1}$. Simulations show that for $\mathrm{n}$ to be exactly $1 / 2$ the base angle $\phi$ must be 41 degrees with $\beta_{1}=$ 1.90 .

We next develop a model for the growth of the cone height with time. The liquid metal in the molten cap is under negative pressure from the $\mathrm{E}^{2}$ force per unit area, $\mathrm{F}_{\mathrm{A}}$, pulling on the surface. This force also acts at the junction between the cap and the side of the cone, serving to pull the viscous molten metal up the side by means of the ponderomotive force, given by the gradient of $F_{A}$. The average flow velocity of the material follows the expression $v=\varepsilon_{0} E_{S}^{2} d / 8 \eta$, where $\eta$ is the viscosity and $d$ is the thickness of the molten layer. This can be converted to a growth rate in height and hence in $\beta$. With a little algebra, we obtain

$$
\begin{aligned}
& \beta=1.9\left[1-\mathrm{BE}_{0}^{4} \mathrm{~T}\right]^{-1 / 6} \\
& \mathrm{~B}=6 \mathrm{~d}^{2} \varepsilon_{0}^{2} / \alpha \eta \mathrm{r}_{1}
\end{aligned}
$$

where $\mathrm{T}$ is the integrated time (repetition rate times the pulse length, with an initial melting time $\approx 30 \mathrm{~ns}$ subtracted from the pulse length). Note that $\mathrm{E}_{0}{ }^{4} \mathrm{~T}$ is a constant at the singularity, in agreement with experiment [V. S. Dolgashev and S. G. Tantawi, "RF Breakdown in X-Band Waveguides, EPAC2002 (see also SLAC-PUB-10355)]. 


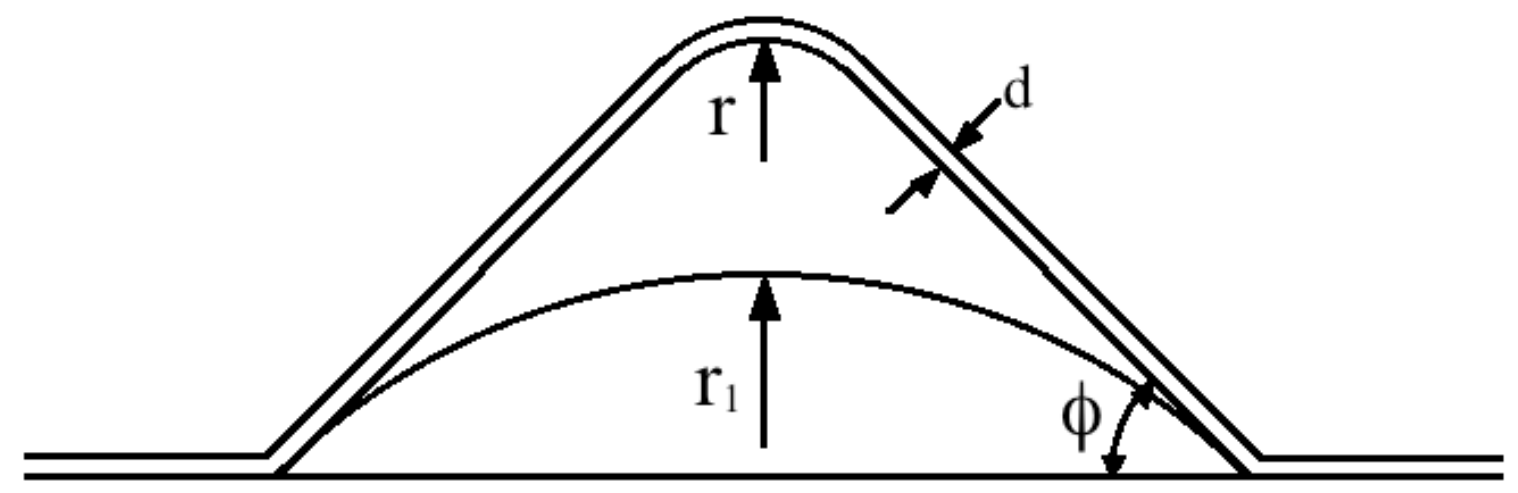

Fig. 2. Geometry modeling the pyramidal features in Fig.1.

Figures of Merit for Metals

\begin{tabular}{|c|c|c|c|}
\hline 1. FM less than 1.0 & 2. $F M=1.00-1.09$ & 3. $F M=1.10-1.19$ & 4. $F M=1.20-1.29$ \\
\hline $\begin{array}{ll}\mathrm{Au} & 0.57\end{array}$ & $\begin{array}{ll}\mathrm{Cu} & 1.00\end{array}$ & $\begin{array}{ll}\mathrm{Zr} & 1.12\end{array}$ & $\begin{array}{ll}\mathrm{Mn} & 1.20\end{array}$ \\
\hline 0.62 & 1.01 & 1.14 & 1.24 \\
\hline 0.74 & 1.02 & 1.15 & 1.25 \\
\hline 0.75 & 1.04 & 1.19 & 1.26 \\
\hline 0.88 & 1.04 & & $\mathrm{Mg} \quad 1.26$ \\
\hline 0.88 & 1.05 & & Co \\
\hline 0.96 & $\mathrm{Rh}$ & & 1.29 \\
\hline Os & & & SS $\quad 1.29$ \\
\hline 5. $\mathrm{FM}=1.43-1.50$ & 6. $\mathrm{FM}=2.28$ & 7. Wildcards & \\
\hline $\begin{array}{ll}\mathrm{Cr} & 1.43\end{array}$ & $\begin{array}{ll}\mathrm{Be} & 2.28^{*}\end{array}$ & $\begin{array}{ll}\mathrm{Si} & 1.54\end{array}$ & \\
\hline 1.45 & & 2.26 & \\
\hline 1.47 & & $2.28 *$ & \\
\hline $\mathrm{V} \quad 1.50$ & & & \\
\hline $\begin{array}{l}\text { *Adjusted for high } \\
\text { Debye temperature }\end{array}$ & & & \\
\hline
\end{tabular}




\section{Periodic table of the elements}

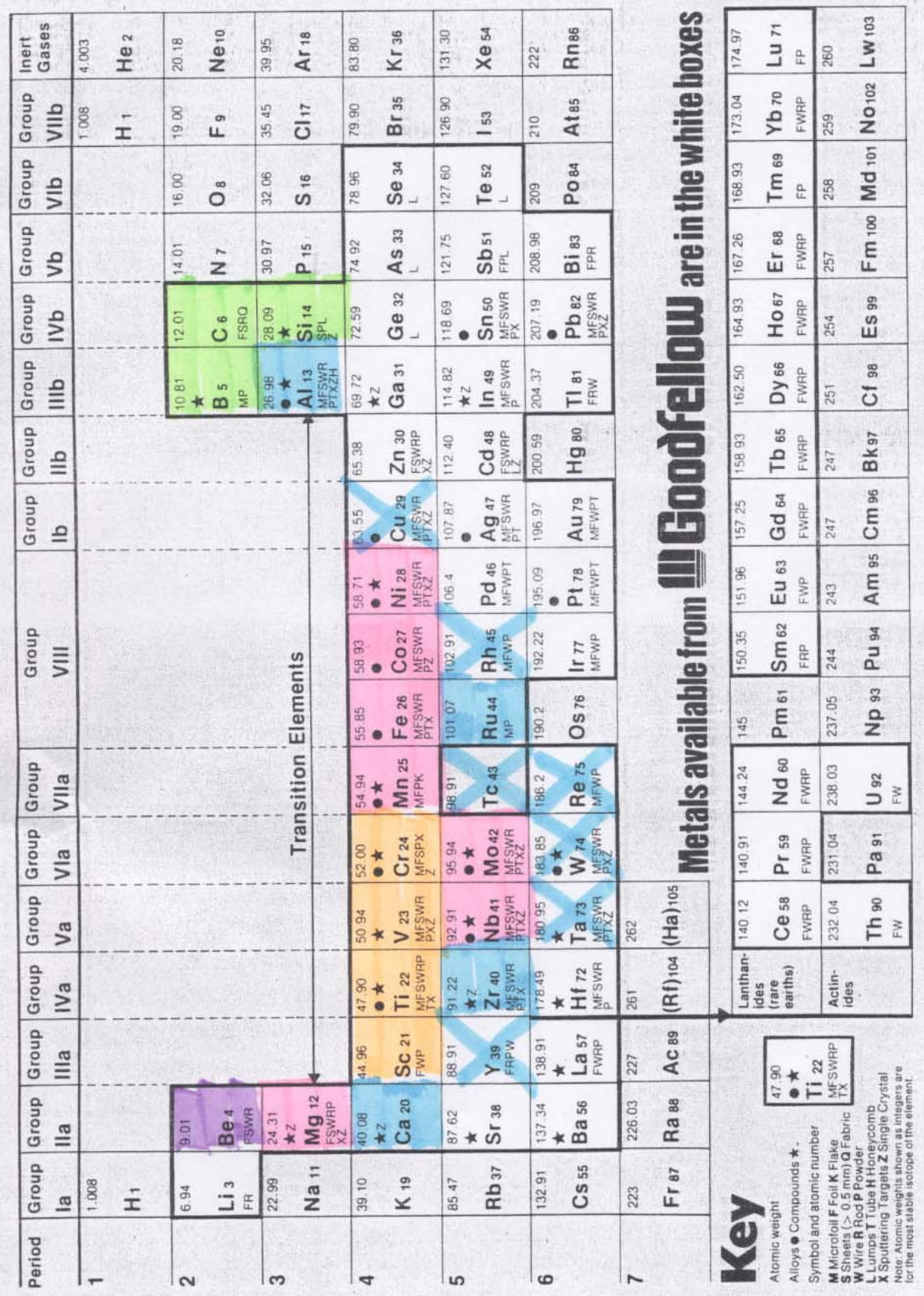

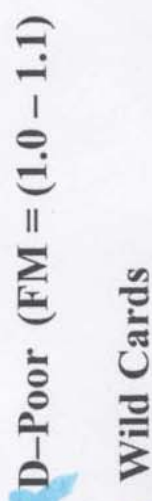

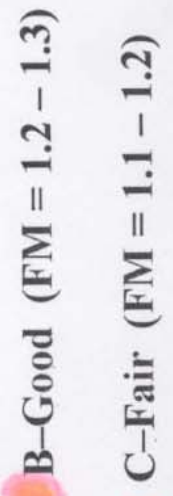

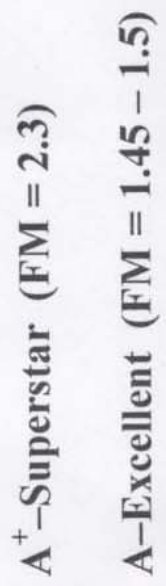




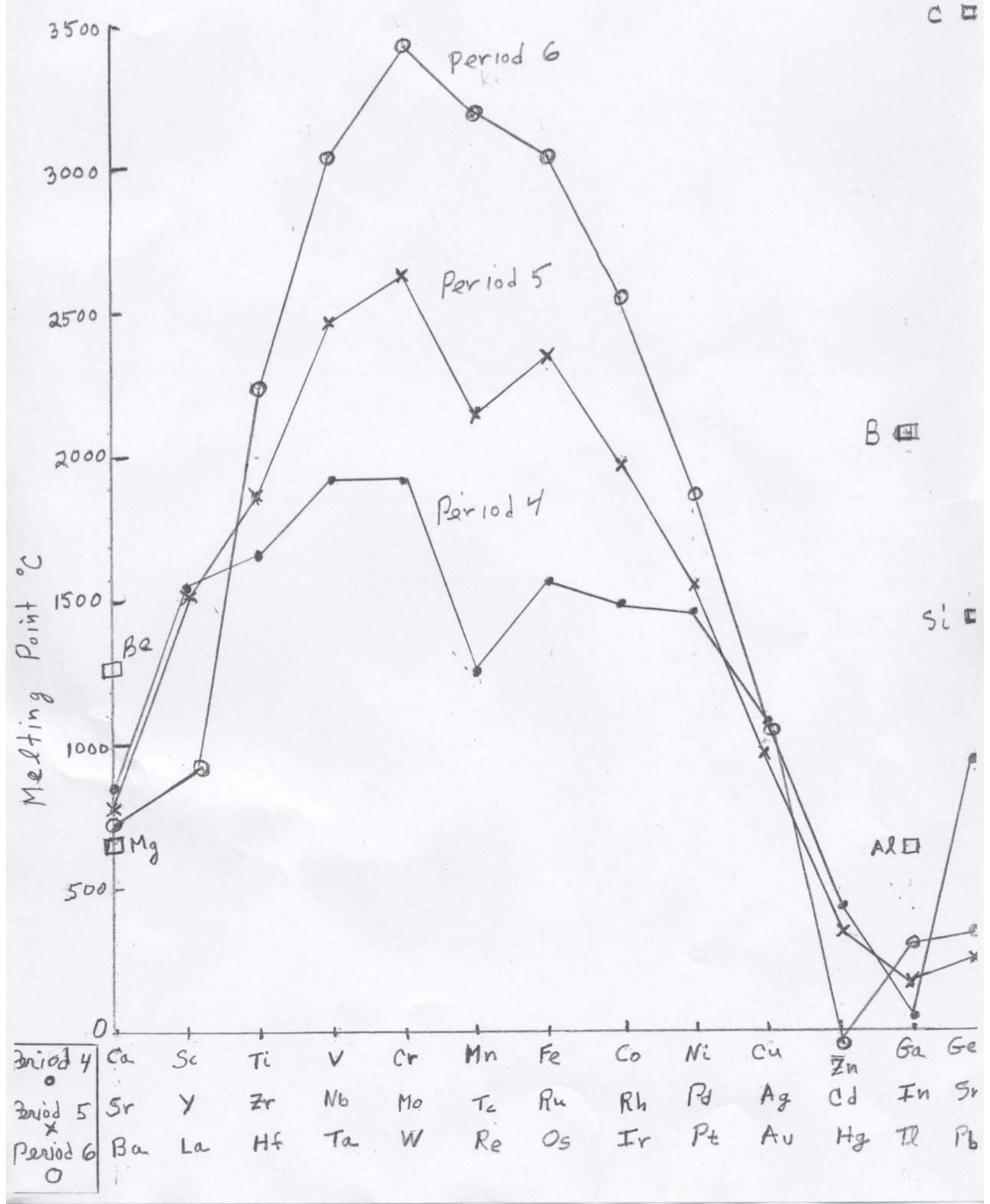

